\title{
The evolution of productivity performance on China's dairy farms in the new millennium
}

\author{
H. Ma, ${ }^{\star 1}$ L. Oxley,† A. Rae, $¥$ C. Fan,§ J. Huang,\# and S. Rozellell \\ ${ }^{*}$ College of Economics and Management, Henan Agricultural University, Zhengzhou 450002, China \\ †Department of Economics and Finance, University of Waikato, Hamilton 3105, New Zealand \\ ‡Department of Economics and Finance, Massey University, Palmerston North 4442, New Zealand \\ $\S$ Agricultural Division, Beijing Municipal Bureau of Finance, Beijing 100037, China \\ \#Center for Chinese Agricultural Policy, IGSNRR, Chinese Academy of Sciences, Beijing 100101, China \\ IIS-ARARC, Freeman Spogli Institute, Stanford University, Stanford 94305-6055, CA
}

\begin{abstract}
China's dairy farm structure has experienced fundamental changes across farm types. As the number of backyard farms has dramatically declined, the share of dairy cows from backyard farms has decreased by $22.4 \%$ from 2003 to 2008 . However, the herd numbers of larger dairy farms have increased. In particular, the share of dairy cows has risen by $18.8 \%$ on small farms, by $22.2 \%$ on medium farms, and by $80.8 \%$ on large farms over the same period. Total factor productivity was decomposed into technical efficiency and technological change on China's dairy farms using the stochastic production frontier framework. The estimated results indicate that patterns of productivity growth appear to have shifted in the 2000s compared with the 1990s, from generally driven by technological change to exclusively driven by technological change on backyard and small farms and uniquely driven by the improvement of technical efficiency on large farms. Tests of the econometric assumption indicate that the variations in total factor productivity growth patterns across farm types and regions are likely caused by the feed input biases and cropping production practice.
\end{abstract}

Key words: China, dairy farm, total factor productivity, technical efficiency

\section{INTRODUCTION}

China's dairy farm structure has experienced fundamental change in both production structure and farm size as the backyard dairy farms (Chinese traditional rural household dairy farms with $<10$ dairy cows; ACPCRD, 2005-2009) dramatically declined and large dairy farms rapidly appeared. For example, the share of dairy cows has decreased by $22.4 \%$ from backyard farms over

Received March 12, 2012.

Accepted July 23, 2012.

${ }^{1}$ Corresponding author: h.y.ma@163.com the period from 2003 to 2008 . However, the herd numbers of dairy cows has risen by $18.8 \%$ on small farms, by $22.2 \%$ on medium farms, and by $80.8 \%$ on large farms over the same period. Therefore, it is interesting to investigate whether productivity performance differs significantly across dairy farm types.

With such changes, we now have the opportunity to observe and analyze the evolution of dairy farm herd size and investigate the productivity performance of dairy farm size. Before 2004, the National Development and Reform Commission (NDRC) collected dairy farm data only for specialized household farms and state-collective farms. However, this survey information system has recently started to provide information on dairy farm production data by farm herd size categorized as follows: backyard dairy farms, small dairy farms, medium dairy farms, and large dairy farms. In particular, the survey now provides information that allows us to investigate the productivity performance of backyard dairy farms, which still account for over $40 \%$ of dairy cattle (CDSY, 2005-2009); these productivity performance data have never been published in English-language economics publications.

Many studies on China's dairy farm productivity performance are available; however, some focus only on technical efficiency (e.g., Peng, 2008), whereas others were conducted in the 1990s and only covered part of the dairy sector (Rae et al., 2006; Ma et al., 2007a,b). Therefore, these studies are unable to reflect on China's dairy farm performance during the new millennium; for example, what does the present productivity growth pattern look like after such fundamental change? Are China's dairy farms still driven by rapid technological change, as was concluded in previous studies?

This study, for the first time, will analyze backyard dairy farms along with the rest of the dairy farms, and results will be reported and classified by herd size. Importantly, our results differ from previous studies and suggest important new policy implications for China's dairy farms. 
In the next section, we describe the new Chinese dairy farm survey data and present some important descriptive statistics. We present a stochastic distance function method to estimate productivity growth, followed by a summary of these new results. In the Discussion, we compare these new results with existing estimates. The paper concludes with some new policy implications from our new findings.

\section{MATERIALS AND METHODS}

\section{Method}

Over the last $20 \mathrm{yr}$, the literature on productivity measurement has developed from the standard indexnumber calculation of total factor productivity (TFP) toward more refined decomposition methods. In the simple TFP framework, the growth rate of the index is usually interpreted as a measure of technical change; however, this interpretation incorporates several restrictive assumptions, such as constant returns to scale and allocative and technical efficiency. More recently, distance functions have been used in an attempt to overcome some of these shortcomings and to identify the components of productivity change (Coelli and Perelman, 2000). This approach does not require any behavioral assumptions, such as cost minimization or profit maximization, to provide a valid representation of the underlying production technology (Brümmer et al., 2002).

For analysis of productivity performance on China's dairy farms, we used a distance function methodology, as this functional form does not require any production behavior assumptions, which might be hard to ascertain given that China's economy is still in a state of transition. Furthermore, we assumed that this input distance function could be approximated by the typical translog functional form. The homogeneity restrictions were imposed by choosing the quantity of one of the inputs as numéraire (a basic standard by which value is computed; here it is assumed to be the number of labor days, $\boldsymbol{X}_{\mathbf{1}}$ ). We then incorporated a farm-type dummy variable into the distance production function to capture the differences across farm types. Second, we incorporated a dairy farm-type dummy variable, concentrate-fodder input ratios $(\boldsymbol{C F R})$, crop production potential $(\boldsymbol{C P P})$, dairy processing capacity $(\boldsymbol{D P C})$, farmer educational level ( $\boldsymbol{E D} \boldsymbol{U})$, and regional dummy variables $\left(\boldsymbol{D}_{\boldsymbol{R}}\right)$ into the technical inefficiency model to observe their effects on technical efficiency. Finally, we incorporated a time variable into the inefficiency model to capture the variations in technical efficiency over time. Details of this type of model and its estimation can be found in Coelli and Perelman (2000), Karagiannis et al. (2004), and Khumbakar and Lovell (2000).

We used the Frontier 4.1 computer program developed by Coelli (1996) to simultaneously estimate the stochastic distance frontier function and technical inefficiency models, as in Coelli and Perelman (2000) and Paul et al. (2000). The input distance function was estimated empirically using pooled panel data for the dairy sector as a whole, because we do not have sufficient observations for each type of dairy farms due to the short sample period (2004-2008). We decomposed productivity growth into technical change (TC) and technical efficiency (TE) components, as in Karagiannis et al. (2004).

\section{Data}

For the period from 1992 to 2003, the national Agricultural Commodity Production Cost and Return Data (ACPCRD) classified dairy farms into only "specialized household dairy farms" and "state-collective dairy farms" and did not produce any production cost information for backyard dairy farms. However, after 2003, the NDRC began to categorize dairy farms into backyard farms ( $\leq 10$ cows), small farms (10 to $\leq 50$ cows), medium farms ( $>50$ to $\leq 500$ cows), and large farms $(>500$ cows $)$, where herd size included lactating and dry cows as well as replacement heifers, and to publish production cost data for each category of dairy farm in China.

Two points should be noted. First, production cost and returns information for backyard dairy farms have only been available since 2003, which allowed us to explore a new research area for China's dairy productivity performance. This is important because backyard dairy farms still account for 30\% of China's total milk production. Second, under the new survey classification system, we were able to observe how dairy farm productivity performed as herd size grew.

The main source of information for examining the productivity of China's dairy farm sector is the ACPCRD, published by the NDRC. The ACPCRD provides detailed output and cost information for a range of farming sectors, including dairy farms in China. Production cost data for dairy farms have rarely been analyzed, especially for the new dairy farm classification system introduced in 2003; the exception would be Peng (2008), who only estimated TE.

The NDRC survey of dairy farm production cost and return data covered approximately 20 major dairy producing provinces (municipal and autonomous regions). Prior to publication, the cost data were summarized in terms of cohorts by averaging similar farms in like 
areas for each observation. The dairy farm production and return cost information was an unbalanced panel of 331 observations for all dairy farms over the period from 2004 to 2008. The panel was unbalanced because the sample distribution was not fixed and part of the sample was normally rotated over some period. Moreover, the dairy farm types varied across province, and some provinces, for example, did not have backyard dairy farms, only medium and large farms (e.g., Beijing, Tianjin, and Shanghai).

Summarizing aspects of these data, Table 1 presents, at the national level over the period from 2004 to 2008, the shares of dairy cow numbers and milk output across 5 cohorts of dairy farm sizes. As can be seen, the share of dairy cow numbers was as high as $56.6 \%$ in 2004 for backyard dairy farms $\left(\boldsymbol{D}_{B}\right)$. This share declined by $13.7 \%$ over the period from 2004 to 2008 . The shares of dairy cow numbers were 23.5, 15.0, and $4.9 \%$ for small dairy farms $\left(\boldsymbol{D}_{S}\right)$, medium dairy farms $\left(\boldsymbol{D}_{M}\right)$, and large dairy farms $\left(\boldsymbol{D}_{\boldsymbol{L}}\right)$ in 2004, respectively. These shares of dairy cow numbers increased to $27.2,19.8$, and $10.1 \%$ in 2008 , respectively. The same pattern can be observed, over time, for the share changes of cow milk output across dairy farm types; in particular, the share increased by over $77 \%$ for large dairy farms.

The first 3 types of dairy farms (backyard, small, and medium) now play a more important role in China's dairy farming sector, whereas large dairy farms contribute little to cow milk production. For example, the first 3 dairy farm types account for $42.9,27.2$, and $19.8 \%$ of total inventory, and for $35.3,28.3$, and $23.0 \%$ of total milk output, respectively. However, large dairy farms only account for $10.1 \%$ of total inventory and $13.3 \%$ of total milk output in China.

From these observations, we can conclude that China's dairy farm structure has experienced fundamental changes at both the national and provincial levels. The share of backyard dairy farms has declined, whereas the share of larger dairy farms has expanded rapidly as the national total cow inventory has grown.

The dairy farm production data discussed above include sufficient information to generate detailed estimates of dairy inputs and outputs on a per-cow basis. The data included milk yield $\left(\boldsymbol{Y}_{1}, \mathrm{~kg}\right)$, by-product value $\left[\boldsymbol{Y}_{2}\right.$, yuan, including calves (male and female), retired animals, and manure], labor inputs ( $\boldsymbol{X}_{1}$, days, including all types of labor), concentrate feed ( $\boldsymbol{X}_{2}$, kilograms, including grain input), fodder consumption $\left(\boldsymbol{X}_{3}\right.$, yuan, deflated by the feed price index), and capital inputs ( $\boldsymbol{X}_{4}$, yuan). For the capital inputs, we used the sum of depreciation, machinery maintenance, and small tool purchases, deflated by the agricultural machinery price index.

\section{The Empirical Model}

To keep the representation of production technology as flexible as possible within the parametric approach, the following function was chosen to approximate the underlying input distance function (Grosskopf et al., 1997; Coelli and Perelman, 2000; Karagiannis et al., 2004):

$$
\begin{aligned}
D_{i t}^{I}= & \alpha_{0}+\sum_{k=1}^{2} \alpha_{k} Y_{k i t}+\sum_{j=1}^{4} \beta_{j} X_{j i t}+\frac{1}{2} \sum_{k=1}^{2} \sum_{l=1}^{2} \alpha_{k l} Y_{k i t} Y_{l i t} \\
& +\frac{1}{2} \sum_{j=1}^{4} \sum_{g=1}^{4} \beta_{j g} X_{j i t} X_{g i t}+\sum_{j=1}^{4} \sum_{k=1}^{2} \delta_{j k} Y_{k i t} X_{j i t}+\sum_{j=1}^{4} \theta_{j} T X_{j i t} \\
& +\sum_{k=1}^{2} \varepsilon_{k} T Y_{k i t}+\gamma T+\eta T^{2}+\sum_{m=1}^{3} \varphi_{m} D_{F m},
\end{aligned}
$$

\begin{tabular}{|c|c|c|c|c|}
\hline Year & Backyard farm & Small farm & Medium farm & Large farm \\
\hline \multicolumn{5}{|l|}{ Share of cow numbers, $\%$} \\
\hline 2004 & 56.6 & 23.5 & 15.0 & 4.9 \\
\hline 2005 & 52.6 & 25.0 & 17.4 & 5.0 \\
\hline 2006 & 51.0 & 25.4 & 17.8 & 5.7 \\
\hline 2007 & 49.0 & 24.6 & 19.0 & 7.4 \\
\hline 2008 & 42.9 & 27.2 & 19.8 & 10.1 \\
\hline Change (2004-2008), \% & -24.2 & 15.7 & 32.0 & 106.1 \\
\hline \multicolumn{5}{|c|}{ Share of cow milk output, $\%$} \\
\hline 2004 & 49.0 & 24.9 & 18.6 & 7.5 \\
\hline 2005 & 44.2 & 27.5 & 20.3 & 7.9 \\
\hline 2006 & 42.5 & 28.0 & 20.8 & 8.7 \\
\hline 2007 & 40.2 & 26.8 & 22.2 & 10.8 \\
\hline 2008 & 35.3 & 28.3 & 23.0 & 13.3 \\
\hline Change (2004-2008), \% & -28.0 & 13.7 & 23.7 & 77.3 \\
\hline
\end{tabular}

Table 1. Evolution of Chinese dairy farm production structure over time ${ }^{1}$ 
where both output and input variables are expressed in natural logarithms; $k, l=1,2$ indicates the type of outputs; $j, g=1,2, \ldots, 4$ indicates the types of inputs; $m=1,2,3$ indicates the types of dairy farms; $i=1,2$, .., $N$ indicates the provinces; $Y_{k i t}$ are outputs and $X_{k i t}$ are inputs as defined in the previous section; $t=1,2$, $\ldots, T$ indicates the annual observations over time, and $\varphi_{x}$ are the parameters to be estimated. The regularity conditions associated with the input distance function require homogeneity of degree 1 in input quantities and symmetry, which implies the following restrictions on the parameters of equation [1]:

$$
\begin{aligned}
& \sum_{k=1}^{2} \beta_{k}=1, \\
& \sum_{j=1}^{4} \beta_{j g}=\sum_{j=1}^{2} \delta_{j k}=\sum_{j=1}^{2} \theta_{i}=0, \\
& \alpha_{k l}=\alpha_{l k}, \text { and } \beta_{j g}=\beta_{g j} .
\end{aligned}
$$

For equation [2], the homogeneity restrictions can be imposed by dividing all input quantities on the righthand side of equation [1] by the quantity of that input as a numeraire $\left(X_{1}\right)$. As in Brümmer et al. (2002), we defined the technical inefficiency term $u_{i t}^{I}$ as a function of time $(\boldsymbol{t})$, concentrate-fodder input ratios $\left(\boldsymbol{C F} \boldsymbol{R}_{i}\right)$, crop production potential $\left(\boldsymbol{C P} \boldsymbol{P}_{i}\right)$, dairy processing capacity $\left(\boldsymbol{D P} \boldsymbol{C}_{\boldsymbol{i}}\right)$, educational level $\left(\boldsymbol{E} \boldsymbol{D} \boldsymbol{U}_{\boldsymbol{i}}\right)$, dairy farm-type dummy $\left(\boldsymbol{D}_{\boldsymbol{F m}}\right)$ and, as Battese and Coelli (1995) argue, some variables may even appear in both functions. Finally, regional dummy variables $\left(\boldsymbol{D}_{\boldsymbol{R} i}\right)$ are included to observe their effects on variations in technical inefficiency:

$$
\begin{aligned}
u_{i t}^{I} & =\varphi_{0}+\varphi_{1} T+\varphi_{2} C F R_{i}+\varphi_{3} C P P_{i}+\varphi_{4} D P C_{i} \\
& +\varphi_{5} E D U_{i}+\sum \varphi_{6 m} D_{F m}+\sum \varphi_{7 i} D_{R i} .
\end{aligned}
$$

Because there are some econometric issues with 2-stage formulation estimation (Khumbakar and Lovell, 2000, p. 264), we used simultaneous estimates of the parameters of equations [1] and [3]. The likelihood function of the model is presented in the appendix of Battese and Coelli (1993). The likelihood function can be expressed in terms of the variance parameters $\sigma^{2}=\sigma_{u}^{2}+\sigma_{v}^{2}$ and $\gamma \equiv \sigma_{u}^{2} / \sigma^{2}$, where $\gamma$ is an unknown parameter to be estimated. We use the Frontier 4.1 computer program developed by Coelli (1996) to estimate the stochastic frontier function and technical inefficiency models simultaneously as in Coelli and Perelman (2000) and Paul et al. (2000).

\section{RESULTS}

All alternative models were statistically significant and thus the translog input distance function, as defined by equation [1], was chosen and the estimated parameters used to conduct further econometric analyses within this study. To test whether the translog was different from the Cobb-Douglas function (MII), whether there were any differences over farm types (MIII, a model to test whether differences existed over farm types), whether technological progress existed (MIV, a model to test whether technological processes existed), whether technological progress was neutral (MV, a model to test whether technological progress was neutral), whether technical efficiency changed over time (MVI, a model to test whether technical efficiency changed over time), and so on, all alternative models were tested and the final likelihood ratio (LR) test statistics are displayed in Table 2. All alternative models were statistically significant and thus the translog input function was valid. The estimated parameters of both the translog input distance function and the inefficiency model were presented in Table 2. The estimated input distance functions for the pooled panel data were found to be well behaved, in that at the point of approximation, it was nonincreasing in outputs and nondecreasing in inputs. The estimated sigma square $\left(\sigma_{u}^{2}=\right.$ $0.116)$ and gamma $(\gamma=0.951)$ were significant at the $1 \%$ level, indicating the presence of technical inefficiency. Thus, a significant part of the output variability among dairy farms could be explained by differences in the degree of TE (Karagiannis et al., 2004).

\section{Econometric Assumption Tests}

Comparing the estimated coefficients (Table 2) with the previous econometric assumptions, we noted the following. The TFP growth pattern on China's dairy farms was feed-input biased, comprising concentrate feed-using and fodder-saving, as the coefficients of $T X_{2}$ and $T X_{3}$ in the input distance function were significantly positive and negative, respectively. This is consistent with our observations, for example, where the concentrate feed input increased by $9.7 \%$ on small farms, $3.1 \%$ on medium farms, and $7.6 \%$ on large farms over the period from 2004 to 2008. On the other hand, fodder input was reduced by $12.8 \%$ on small farms, by $9.6 \%$ on medium farms, and by $11.9 \%$ on large farms over the same period.

The production frontiers were different for backyard and larger dairy farms because the coefficients of $D_{S}$, $D_{M}$, and $D_{L}$ in the input distance function were significantly different from zero. This suggests that significant 
Table 2. The estimates of translog input distance function and inefficiency model for China's dairy farms ${ }^{1}$

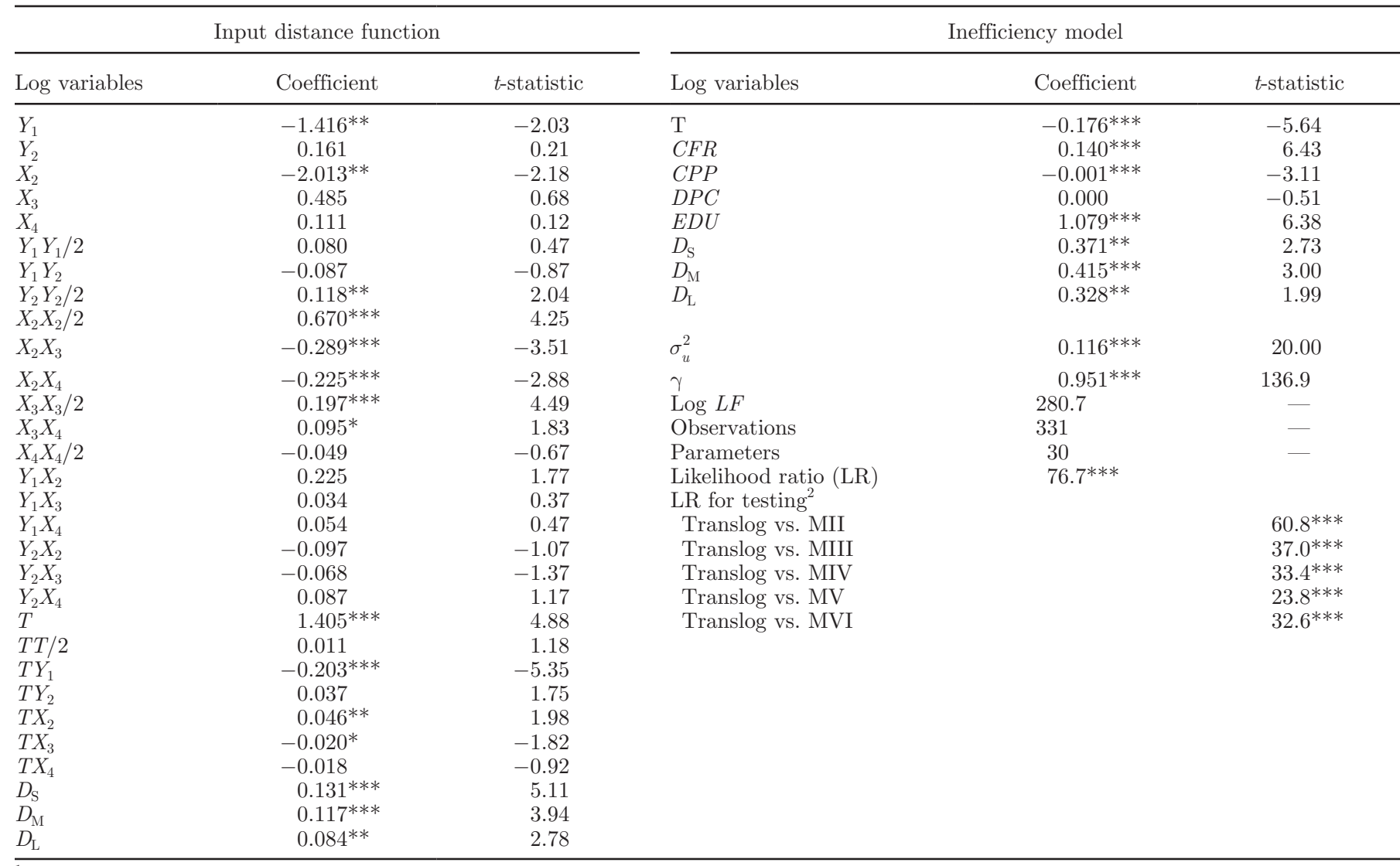

${ }^{1}$ Constant term and provincial dummies in inefficiency model are not displayed. Two-thirds of provincial dummies in the inefficiency model were statistically significant. $X_{1}$ is used as numeraire; $X$ and $Y=$ input and output, and $T=$ time variable; $C F R=$ concentrate-fodder input ratios; $C P P=$ crop production potential; $D P C=$ dairy processing capacity; $E D U=$ educational level;

$D_{\mathrm{S}}=$ small farm dummy; $D_{\mathrm{M}}=$ medium farm dummy; $D_{\mathrm{L}}=$ farm dummy.

${ }^{2} \mathrm{LR}$ for testing is $\chi^{2}$ statistic. MII = Cobb-Douglas function; MIII = model to test whether there were any differences over farm types; MIV $=$ model to test whether technological progress existed; $\mathrm{MV}=$ model to test whether technological progress was neutral; MVI $=$ model to test whether technical efficiency changed over time.

***, ${ }^{* *}$, and $*$ indicate 1,5 , and $10 \%$ significant levels, respectively.

differences existed in the yields between backyard farms and the other 3 types of dairy farms. The average yield was $4,977 \mathrm{~kg}$ on backyard farms, $5,569 \mathrm{~kg}$ on medium farms, and $6,262 \mathrm{~kg}$ on large farms over the period from 2004 to 2008.

Technical efficiency improved over time, because the coefficient on the time variable in the technical inefficiency model was significantly negative, indicating that time $(t)$ had a significant positive effect on improvements in TE. Therefore, in general, TE on China's dairy farms improved over the study period.

It was important to ascertain whether $C F R$ was statistically significant from zero, which would suggest that the concentrate:fodder input ratio has a positive effect on technical inefficiency. In other words, the concentrate:fodder input ratio is significantly negatively associated with TE, implying that farmers should reduce the ratio to improve TE. In fact, the concentrate:fodder input ratio increased over the study period, from 2.75 to 3.46 on small farms, from 1.88 to 2.11 on medium farms, and from 1.50 to 1.83 on large farms.

We expected to find that $C P P$ was significantly negatively associated with technical inefficiency, although in our results the coefficient was small. In other words, we expected that $C P P$ would be positively associated with TFP growth. The results suggest that TFP growth rates were either high or on an increasing trend. In fact, TFP growth rates were faster in most of the major crop-producing regions; for example, in Inner Mongolia, Jilin, Heilongjiang, Shandong, and Henan for backyard farms; in Hebei, Jilin, Heilongjiang, Shandong, and Henan for small farms; and in Inner Mongolia, Jilin, and Heilongjiang for medium farms. 
Table 3. Decomposition of total factor productivity (TFP) into technical efficiency (TE) and technological change (TC) over dairy farm types at the national level in China from 2004 to 2008

\begin{tabular}{lcrcr}
\hline & & \multicolumn{3}{c}{ TFP decomposition, \% } \\
\cline { 3 - 5 } Dairy & Observations, no. & TFP & TE & TC \\
\hline farm type & 69 & 3.14 & 0.35 & 2.79 \\
Backyard & 81 & 3.20 & 0.92 & 2.28 \\
Small & 97 & 0.52 & 0.75 & -0.23 \\
Medium & 84 & -1.76 & 1.00 & -2.76 \\
Large & & &
\end{tabular}

The estimated results showed that $D P C$ was nonsignificantly associated with technical inefficiency. However, $E D U$ was significantly positively associated with technical inefficiency, which suggests that education levels were negatively correlated with TE on China's dairy farms, as estimated by this study. This result seems counterintuitive, but appears to be common for China. For example, Tian and Wan (2000) report no consistent relationship between the education variable and TE. As Tian and Wan (2000) argued, this inconsistency might be related to the fact that schooling is not an appropriate indicator of the knowledge and skills needed or utilized in actual farm management in rural China. Chen and Wan (1997) even argue it is more likely that laborers with higher education work off-farm, especially in the economically developed and rice-producing southern China.

To consider the results in more detail, the estimated parameters of the input distance function were used to estimate growth rates of TC and TE improvement, and the TFP growth rate was constructed by summing TC and TE improvement. The TFP decompositions are presented as Tables 3, 4, 5, 6, and 7, and the TE levels are displayed in Table 8. From these estimated results, we present the following empirical analyses.

\section{Productivity Growth Across Farm Types}

Table 3 shows apparent variations in productivity growth rates and growth pattern over various farm types; for example, the annual TFP growth rates were high on backyard farms and small farms, 3.14 and $3.20 \%$, respectively. However, the annual TFP growth rates on medium farms and large farms were only 0.52 and $-1.76 \%$, respectively. The TFP decomposition analysis clearly shows that TC, not improvements to efficiency, was the driver of the increase in productivity on China's dairy farms. For example, TC accounted for nearly $90 \%$ of total productivity growth on backyard farms and for $70 \%$ on small farms. Technological deterioration was the only source of decline in productivity for large farms.

\section{Regional Backyard Dairy Farms}

The estimated results show that regional backyard farms followed different patterns of TFP growth. For example, some provinces had positive growth rates of TC, whereas others had negative growth rates of TE (Table 4). This resulted in an apparent variation in TFP growth rate over regions. The highest value of

Table 4. Regional decomposition of total factor productivity (TFP) into technical efficiency (TE) and technological change (TC) on backyard dairy farm in China

\begin{tabular}{|c|c|c|c|c|c|}
\hline \multirow[b]{2}{*}{ Province $^{1}$} & \multirow[b]{2}{*}{ Period } & \multirow{2}{*}{$\begin{array}{c}\text { Observations, } \\
\text { no. }\end{array}$} & \multicolumn{3}{|c|}{ TFP decomposition, $\%$} \\
\hline & & & TFP & $\mathrm{TE}$ & $\mathrm{TC}$ \\
\hline Shanxi & 2004-2008 & 5 & 0.30 & -0.41 & 0.70 \\
\hline Inner Mongolia & 2004-2008 & 5 & 4.64 & 0.22 & 4.42 \\
\hline Jilin & 2004-2008 & 5 & 4.84 & 1.08 & 3.76 \\
\hline Heilongjiang & 2004-2008 & 4 & 4.16 & 2.37 & 1.79 \\
\hline Zhejiang & 2004-2006 & 3 & 1.47 & -1.23 & 2.70 \\
\hline Shandong & 2004-2008 & 5 & 2.11 & -0.17 & 2.28 \\
\hline Henan & 2004-2008 & 5 & 6.19 & 2.97 & 3.22 \\
\hline Guangxi & 2004-2008 & 5 & 1.38 & 1.30 & 0.08 \\
\hline Chongqing & 2004-2008 & 5 & 0.08 & 0.22 & -0.14 \\
\hline Guizhou & $2005-2008$ & 4 & 0.85 & -0.34 & 1.18 \\
\hline Yunnan & $2004-2007$ & 4 & 10.95 & 1.49 & 9.46 \\
\hline Shaanxi & 2004-2008 & 5 & -2.11 & -7.45 & 5.34 \\
\hline Xinjiang & 2004-2008 & 5 & 3.76 & 0.79 & 2.97 \\
\hline
\end{tabular}

${ }^{1}$ Only those provinces with $\geq 3$ observations are listed. 
Table 5. Regional decomposition of total factor productivity (TFP) into technical efficiency (TE) and technological change (TC) on small dairy farm in China

\begin{tabular}{|c|c|c|c|c|c|}
\hline \multirow[b]{2}{*}{ Province $^{1}$} & \multirow[b]{2}{*}{ Period } & \multirow{2}{*}{$\begin{array}{c}\text { Observations, } \\
\text { no. }\end{array}$} & \multicolumn{3}{|c|}{ TFP decomposition, $\%$} \\
\hline & & & TFP & $\mathrm{TE}$ & $\mathrm{TC}$ \\
\hline Tianjin & 2004-2008 & 5 & -5.78 & -7.79 & 2.01 \\
\hline Hebei & 2004-2008 & 5 & 3.23 & 1.14 & 2.09 \\
\hline Shanxi & $2006-2008$ & 3 & 0.99 & -0.94 & 1.94 \\
\hline Inner Mongolia & 2004-2008 & 5 & 1.26 & -2.86 & 4.12 \\
\hline Liaoning & $2004-2008$ & 5 & -1.73 & -0.46 & -1.27 \\
\hline Jilin & 2004-2008 & 5 & 3.31 & 0.68 & 2.63 \\
\hline Heilongjiang & 2004-2008 & 5 & 6.26 & 5.08 & 1.18 \\
\hline Fujian & $2004-2008$ & 5 & 3.15 & 4.92 & -1.77 \\
\hline Shandong & 2004-2008 & 5 & 5.02 & 1.67 & 3.36 \\
\hline Henan & $2004-2008$ & 5 & 1.30 & -0.77 & 2.07 \\
\hline Hunan & 2004-2008 & 5 & 11.82 & 5.72 & 6.10 \\
\hline Guangxi & $2004-2008$ & 5 & 6.20 & 5.80 & 0.39 \\
\hline Sichuan & 2004-2008 & 5 & 3.52 & 1.69 & 1.82 \\
\hline Yunnan & 2004-2008 & 5 & 7.62 & 0.17 & 7.46 \\
\hline Ningxia & $2004-2008$ & 4 & -0.42 & -0.10 & -0.32 \\
\hline Xinjiang & 2004-2007 & 3 & 2.06 & 0.19 & 1.87 \\
\hline
\end{tabular}

${ }^{1}$ Only those provinces with $\geq 3$ observations are listed.

TC was found in Yunnan, with an annual growth rate of 9.46\%. In Inner Mongolia, Jilin, and Shaanxi, the growth of TC ranged from 3.70 to $5.30 \%$. The growth of TC was slower in Heilongjiang, Zhejiang, Shandong, Henan, and Xinjiang, ranging from approximately 2.00 to $3.00 \%$ annually. Technological change was static in Shanxi and Guangxi and worsened in Chongqing, where the growth rate was $-0.14 \%$. Similar patterns emerge for TE. As a result, productivity was best in Yunnan with a $10.95 \%$ growth rate annually; productivity was good in Inner Mongolia, Jilin, Heilongjiang, and Henan, where TFP growth rates ranged from 4.00 to $6.20 \%$; and productivity growth was slowest in Shanxi, Chongqing, and Guizhou, where the TFP changed little ( $<1 \%$ annually). Productivity actually worsened in Shaanxi, where the TFP growth rate was $-2.11 \%$. It appears that TC enhanced TFP growth, whereas falling TE apparently offset this effect on TFP growth in some cases (e.g., in Zhejiang and Shaanxi).

However, although TC and TE showed unbalanced growth and most of the TFP growth was driven by TC, in some provinces, TFP growth arose due to both

Table 6. Regional decomposition of total factor productivity (TFP) into technical efficiency (TE) and technological change (TC) on medium dairy farm in China

\begin{tabular}{|c|c|c|c|c|c|}
\hline \multirow[b]{2}{*}{ Province $^{1}$} & \multirow[b]{2}{*}{ Period } & \multirow{2}{*}{$\begin{array}{c}\text { Observations, } \\
\text { no. }\end{array}$} & \multicolumn{3}{|c|}{ TFP decomposition, $\%$} \\
\hline & & & TFP & $\mathrm{TE}$ & $\mathrm{TC}$ \\
\hline Beijing & 2004-2008 & 5 & 1.52 & 0.60 & 0.92 \\
\hline Tianjin & 2004-2008 & 5 & -0.58 & -3.12 & 2.54 \\
\hline Shanxi & $2006-2008$ & 3 & 1.78 & -1.58 & 3.35 \\
\hline Inner Mongolia & 2004-2008 & 5 & 1.36 & 0.00 & 1.36 \\
\hline Jilin & 2004-2008 & 5 & 12.16 & 10.53 & 1.64 \\
\hline Heilongjiang & 2004-2008 & 5 & 4.86 & 4.98 & -0.12 \\
\hline Shanghai & 2004-2008 & 5 & -3.88 & 2.34 & -6.22 \\
\hline Zhejiang & $2004-2006$ & 3 & -2.92 & -0.47 & -2.45 \\
\hline Anhui & 2004-2008 & 5 & 1.47 & 0.40 & 1.08 \\
\hline Fujian & 2004-2008 & 5 & 0.43 & 2.87 & -2.44 \\
\hline Henan & 2004-2008 & 5 & 0.58 & -0.31 & 0.89 \\
\hline Hunan & 2004-2008 & 4 & -4.12 & -2.59 & -1.53 \\
\hline Guangxi & 2004-2008 & 5 & 13.37 & 11.78 & 1.60 \\
\hline Hainan & 2004-2006 & 3 & 2.46 & 0.54 & 1.92 \\
\hline Chongqing & 2004-2008 & 5 & -0.78 & -0.51 & -0.27 \\
\hline Shaanxi & 2004-2008 & 5 & -3.75 & 0.38 & -4.13 \\
\hline Gansu & 2004-2008 & 5 & -0.54 & -1.47 & 0.93 \\
\hline Ningxia & 2004-2008 & 5 & 9.89 & 9.25 & 0.64 \\
\hline Xinjiang & $2005-2008$ & 4 & -1.13 & 0.18 & -1.31 \\
\hline
\end{tabular}

${ }^{1}$ Only those provinces with $\geq 3$ observations are listed. 
Table 7. Regional decomposition of total factor productivity (TFP) into technical efficiency (TE) and technological change (TC) on large dairy farm in China

\begin{tabular}{llcrrr}
\hline & & & \multicolumn{3}{c}{ TFP decomposition, \% } \\
\cline { 3 - 6 } Province $^{1}$ & Period & $\begin{array}{c}\text { Observations, } \\
\text { no. }\end{array}$ & TFP & TE & TC \\
\hline Beijing & $2004-2008$ & 3 & -6.88 & 0.06 & -6.95 \\
Tianjin & $2004-2008$ & 5 & -6.12 & -1.60 & -4.52 \\
Liaoning & $2004-2008$ & 5 & -1.21 & 0.47 & -1.68 \\
Heilongjiang & $2004-2008$ & 5 & 2.51 & 1.49 & 1.02 \\
Jiangsu & $2004-2008$ & 5 & -5.72 & 0.68 & -6.40 \\
Zhejiang & $2004-2008$ & 5 & -4.82 & -0.27 & -4.55 \\
Anhui & $2004-2008$ & 5 & -2.46 & -0.18 & -2.28 \\
Fujian & $2004-2007$ & 4 & 1.27 & 3.44 & -2.17 \\
Shandong & $2004-2008$ & 5 & -4.03 & 0.32 & -4.35 \\
Henan & $2004-2008$ & 5 & -0.57 & 0.25 & -0.82 \\
Hubei & $2004-2008$ & 5 & 0.28 & 2.97 & -2.69 \\
Guangdong & $2004-2008$ & 5 & 0.94 & -0.39 & 1.34 \\
Gansu & $2004-2008$ & 5 & -4.27 & 1.28 & -5.54 \\
Qinghai & $2004-2008$ & 5 & 2.51 & -0.11 & 2.61 \\
Xinjiang & $2004-2008$ & 5 & -5.92 & 0.48 & -6.40 \\
\hline
\end{tabular}

${ }^{1}$ Only those provinces with $\geq 3$ observations are listed.

TC and TE. For example, in Heilongjiang, the growth rate was $2.37 \%$ for $\mathrm{TE}$, and $1.79 \%$ for TC annually. Similarly, in Henan, the growth rate was $3.22 \%$ for TC and $2.97 \%$ for TE. Clearly, falling TE, in most cases, offset the effect of rapid technological change on TFP growth, resulting in modest TFP growth (e.g., as in Zhejiang and Shaanxi provinces).

We observed 2 extreme cases, where TC was extremely fast for one province, and TE improved extremely rapidly in another province. The growth rate of TC was as high as $9.46 \%$ annually in Yunnan, whereas the growth rate of TE was as low as $-7.45 \%$ in Shaanxi. It is clear that improvements to TE should be the priority of scientific and technological policies applied to dairy farming. Meanwhile, more attention should be given to Yunnan, where its high growth rate of TC should act as an exemplar.

\section{Regional Small Dairy Farms}

Variations in TFP growth rates were apparent across locations, as some regions showed rapid TFP growth whereas others had large negative TFP growth rates (Table 5). For example, productivity was best in $\mathrm{Hu}-$ nan, where the TFP growth rate was as high as $11.82 \%$ annually; next were Heilongjiang, Shandong, Guangxi, and Yunnan provinces, where TFP growth rates ranged from 5 to $7 \%$. The third group included Hebei, Jilin, Fujian, and Sichuan, where TFP growth rates were approximately $3 \%$. Productivity actually worsened in Liaoning and Ningxia provinces, where TFP growth rates were -1.73 and $-0.42 \%$, respectively. The worst performance was in Tianjin, where TFP decreased by $5.78 \%$ annually.
It appeared that TFP growth was driven by improvements in TE in some regions (e.g., Heilongjiang, Fujian, Hunan, and Guangxi), whereas it was driven by rapid TC in others (e.g., Inner Mongolia, Jilin, Shandong, and Yunnan). However, TFP growth appeared to come from both TE and TC; for example, in Hunan, TE improved rapidly ( $5.72 \%$ annually) and TC was also rapid (6.10\% annually), suggesting that both played similar roles in the TFP growth in this province. The same can be said of Hebei and Sichuan, although their TFP growth rates $(\sim 3.3 \%)$ were much slower than those in Hunan.

Some outliers were found in terms of TE and TC; for example, the largest negative TE was found in Tianjin, where the rate was as low as $-7.79 \%$, whereas the fastest growth of TC was found in Yunnan, where the growth rate was as high as $7.46 \%$. Other extreme cases can be seen for the improvements in TE, which was as fast as 5\% annually in Heilongjiang, Fujian, Hunan, and Guangxi. However, many provinces remained static for both TE and TC; for example, Yunnan, Ningxia, and Xinjiang had almost unchanged TE, whereas Guangxi and Ningxia had negligible TC over time.

\section{Regional Medium Dairy Farms}

As found in the previous analysis presented above, we observed variations in TFP growth rates across regions: some displayed large positive TFP growth, and others large negative TFP growth (Table 6). For example, the TFP growth rates were as high as $12.16 \%$ in Jilin, $13.37 \%$ in Guangxi, $9.89 \%$ in Ningxia, $4.86 \%$ in Heilongjiang, and $2.46 \%$ in Hainan. However, productivity declined almost as fast in some provinces: $3.88 \%$ in 
Table 8. Technical efficiency level (\%) across dairy farm types and over time

\begin{tabular}{|c|c|c|c|}
\hline Farm type/province ${ }^{1}$ & 2004-2008 & 2004 & 2008 \\
\hline \multicolumn{4}{|l|}{ Backyard dairy farms } \\
\hline National & 96 & 95 & 97 \\
\hline Shanxi & 95 & 97 & 95 \\
\hline Inner Mongolia & 92 & 92 & 93 \\
\hline Jilin & 93 & 91 & 95 \\
\hline Heilongjiang & 94 & 89 & 96 \\
\hline Zhejiang & 96 & 97 & - \\
\hline Shandong & 95 & 96 & 95 \\
\hline Henan & 87 & 81 & 92 \\
\hline Guangxi & 88 & 86 & 90 \\
\hline Yunnan & 96 & 94 & - \\
\hline Shaanxi & 86 & 97 & 72 \\
\hline Xinjiang & 97 & 94 & 97 \\
\hline \multicolumn{4}{|l|}{ Small dairy farms } \\
\hline National & 94 & 91 & 95 \\
\hline Tianjin & 79 & 97 & 71 \\
\hline Hebei & 89 & 84 & 88 \\
\hline Shanxi & 91 & - & 91 \\
\hline Inner Mongolia & 89 & 96 & 86 \\
\hline Liaoning & 97 & 98 & 96 \\
\hline Jilin & 94 & 90 & 93 \\
\hline Heilongjiang & 90 & 78 & 95 \\
\hline Shandong & 94 & 89 & 95 \\
\hline Henan & 92 & 92 & 90 \\
\hline Sichuan & 86 & 81 & 86 \\
\hline Yunnan & 96 & 97 & 98 \\
\hline Ningxia & 97 & 95 & 96 \\
\hline Xinjiang & 97 & 97 & 97 \\
\hline \multicolumn{4}{|l|}{ Medium dairy farms } \\
\hline National & 95 & 93 & 96 \\
\hline Beijing & 96 & 95 & 97 \\
\hline Tianjin & 86 & 95 & 84 \\
\hline Shanxi & 89 & - & 89 \\
\hline Inner Mongolia & 97 & 97 & 97 \\
\hline Jilin & 89 & 63 & 96 \\
\hline Heilongjiang & 92 & 78 & 95 \\
\hline Henan & 94 & 95 & 94 \\
\hline Guangxi & 83 & 58 & 93 \\
\hline Yunnan & 98 & 97 & 98 \\
\hline Shaanxi & 95 & 94 & 95 \\
\hline Gansu & 93 & 97 & 92 \\
\hline Ningxia & 89 & 65 & 94 \\
\hline Xinjiang & 96 & 97 & 98 \\
\hline \multicolumn{4}{|l|}{ Large dairy farms } \\
\hline National & 95 & 93 & 96 \\
\hline Beijing & 96 & 94 & 96 \\
\hline Tianjin & 93 & 96 & 90 \\
\hline Liaoning & 96 & 94 & 96 \\
\hline Heilongjiang & 96 & 91 & 97 \\
\hline Jiangsu & 79 & 75 & 77 \\
\hline Zhejiang & 96 & 97 & 96 \\
\hline Shandong & 97 & 96 & 97 \\
\hline Henan & 96 & 94 & 95 \\
\hline Gansu & 95 & 92 & 97 \\
\hline Qinghai & 96 & 96 & 96 \\
\hline Xinjiang & 97 & 95 & 97 \\
\hline
\end{tabular}

${ }^{1}$ Only those provinces with $\geq 3$ observations are listed.

Shanghai, $4.12 \%$ in Hunan, and $3.75 \%$ in Shaanxi, and TFP growth remained static, around 1.5\% annually, for many regions (e.g., Beijing, Shanxi, Inner Mongolia, Anhui). In some regions, productivity performance actually deteriorated; for example, Tianjin $(-0.58 \%)$, Chongqing $(-0.78 \%)$, and Xinjiang $(-1.13 \%)$.

The TFP growth patterns for this group were mixed, with significant variations in both TE and TC across regions. For example, some regions displayed a large positive growth rate of TE improvement; for example, Jilin (10.53\%), Guangxi (11.78\%), and Ningxia (9.89\%), whereas others showed large negative growth rate of TE improvement; for example, Tianjin $(-3.12 \%)$ and Hunan (-2.59\%). Furthermore, some regions showed a large positive growth rate of TC; for example, Tianjin $(2.54 \%)$ and Shanxi $(3.35 \%)$, whereas others showed a large negative growth rate of TC; for example, Shanghai $(-6.22 \%)$ and Shaanxi $(-4.13 \%)$. Therefore, we might conclude that, on China's medium dairy farms, both TFP growth rates and growth patterns varied significantly across regions. The productivity performance on medium dairy farms showed considerable regional diversity, where some of the variation was driven by TE (e.g., in Jilin, Heilongjiang, and Ningxia) and some by TC (e.g., in Tianjin, Shanxi, and Hainan).

Various extreme and special cases can be seen in the results for TFP growth, TE, and TC. For example, all 3 growth rates were stable and stagnant for certain regions (e.g., Henan and Chongqing), some regions had extremely high growth in TE (e.g., Jilin, Gansu, and Ningxia, around 10\%), and other regions were faced with a rapid deterioration of TC (e.g., Shanghai and Shaanxi). More stable cases can be found for each of the 3 individual growth rates. Therefore, to enhance productivity and improve TE on China's medium dairy farms, these extreme and special cases should be given particular attention, as they played a critical role in the productivity performance on China's medium dairy farms.

\section{Regional Large Dairy Farms}

Regional large farms had many more large negative TFP growth rates than positive large growth rates (Table 7). For example, the TFP growth rates ranged from -4.03 to $-6.88 \%$ in many regions (e.g., Beijing, Tianjin, Jiangsu, Zhejiang, Shandong, Gansu, and Xinjiang), whereas only 3 regions had TFP growth rates over 1.00\% (Heilongjiang, Fujian, and Qinghai). In addition, productivity appeared to be almost static in the remaining regions. Thus, it seems that the productivity performance was homogeneous for a large number of regions when the focus was on large dairy farms. Moreover, the performance of large farms, compared with other 3 types of dairy farms, suggests that these large farms display the worse productivity performance in China's dairy sector. 
TE

Table 8 presents the levels of TE by type of dairy farm and location. As can be seen, the average levels of TE increased over the study period. On backyard dairy farms, TE increased from 0.95 in 2004 to 0.97 in 2008; on small dairy farms, it increased from 0.91 to 0.97; on medium dairy farms, it increased from 0.93 to 0.96 ; and on large dairy farms, it increased from 0.93 to 0.96 . These observations are consistent with the estimates of TE presented in previous studies. In 2008, the lowest TE of backyard farms was $72 \%$, in Shaanxi, and the highest 97\%, in Beijing and Xinjiang. For small dairy farms, the lowest TE was in Tianjin (71\%) and the highest was in Yunnan (98\%). For medium dairy farms, the lowest TE was in Tianjin (84\%) and the highest was in Yunnan and Xinjiang (98\%). For large dairy farms, the lowest TE was in Jiangsu (77\%) and the highest in Gansu and Xinjiang (97\%).

Although most of the regional TE have improved since 2004, a few regional TE have deteriorated; for example, on backyard dairy farms in Shaanxi, on small dairy farms in Tianjin and Inner Mongolia, on medium dairy farms in Tianjin and others, and on large dairy farms in Tianjin. However, TE apparently improved on backyard dairy farms in Heilongjiang, Henan, and Guangxi; on small dairy farms in Heilongjiang and Shandong; on medium dairy farms in Jilin, Heilongjiang, Guangxi, and Ningxia; and on large dairy farms, again in Heilongjiang. Therefore, the patterns of TE varied somewhat across the regions.

\section{DISCUSSION}

Given the rapid evolution of China's dairy farm sector, it is not surprising that the dynamism of China's dairy farm sector can be seen in our analysis. When we track the recent trends in dairy herd cohorts, we can see what appears to be a rapidly shifting pattern in dairy farm structure. Instead of increasing backyard farms, after 2003, new dairy herd growth occurred in the larger dairy units, although backyard farms still account for approximately $35 \%$ of the total farm sector. However, as argued by Wang (2005), this change in dairy production structure created a huge challenge for policy-makers in regard to how to set new development policies. In the new millennium, China's dairy farms are faced with many new challenges (Li, 2007), including switches from dairy to beef cattle in some regions ( $\mathrm{Hu}, 2007)$.

The pattern of productivity growth on dairy farms has several policy implications. The present dairy production practice, which is concentrate feed-using and fodder-saving, is inconsistent with the national policy, called "Guo Fu Huan Tian," which means "crop straw for animals and then excrement for the land." The concentrate-using and fodder-saving input biases also do not coincide with China's agricultural output situation. More importantly, perhaps, such dairy farming practices are causing serious underconsumption of fodder by dairy cattle, resulting in a failure to meet their physiological demands for fodder. This might result in slow productivity growth on China's dairy farm. Therefore, we conclude that the present fodder input level is significantly lower than that found in other developed countries and below the normal physiological demands of the cows. As a result, fodder cellulose supply could not maintain the normal functioning of the rumen, and low fodder input results in the shortage of both energy and the synthetic materials for milk and therefore reducing milk yield (Fu and Zhang, 2009). Normally, herbage accounts for $61 \%$ of total feed input in the United States, whereas the ratio of concentrate feed to fodder was 45:55 in China (Hou, 2006). However, in China, the average ratios of concentrate feed to herbage were $62: 38$ for large dairy farms, 66:34 for medium dairy farms, and higher for small and backyard dairy farms over the 2004-2008 period.

Fodder quality can be another important factor for dairy farm productivity. Concerned by the high price of alfalfa and rising production costs, dairy farms use little alfalfa in the daily fodder ration, and many backyard farmers used no alfalfa at all (Hou, 2006). In fact, only large, urban dairy farms use alfalfa, whereas the large cropping areas still use crop straw (e.g., corn, wheat, and peanut straw in the north; rice straw in the south) as the major fodder sources ( $\mathrm{Li}$ et al., 2007). Thus, many Chinese dairy experts conclude that reducing sown areas and output of alfalfa would not meet dairy farm demand in China (Yang and Li, 2009), where the quantity and the quality of fodder has seriously impeded the development of dairy production (Liu, 2009; PREC, 2009; Yang, 2009; Zhang et al., 2010; Chen, 2012). No standard dairy daily fodder ration exists in China; it depends upon local crop by-products. Because of regional variations in crop production, regional differences exist in dairy daily fodder rations. Typically, backyard farms use local peanut and soybean straw, and corn and wheat straw as fodder sources, and some use tofukasu and brewers grains in the daily fodder ration. Small farms typically use half peanut straw and half corn or wheat straw in the daily fodder ration, whereas large farms use half alfalfa or Leymus chinensis (Chinese lyme grass) and half silage in the daily fodder ration. However, it should be noted that alfalfa or Leymus chinensis inputs account for only $50 \%$ of 
total fodder on some large farms. Furthermore, in some regions, alfalfa needs to be purchased and most of this is imported into China.

Why have larger farms fallen behind in terms of technology use and adoption? It is perhaps not surprising to find that the technologies on large dairy farms were all current when large dairy farms were established in the new millennium. Some large commercial dairy farms (including dairy cattle and facilities) were imported "as a whole." As a result, they did not need to, and could not, adopt any other new dairy farm technologies within a short period. Therefore, the technologies on large dairy farms gradually declined over some fixed period, which perhaps coincides with our study period. In other words, it appears that large dairy farms have faced a serious challenge to maintain or adopt modern technologies in China. In fact, the cost data show that the yield of large dairy farms increased by only $1.6 \%$, but concentrate feed input increased by $7.6 \%$ over the 2004 to 2008 period. As a result, the output-input ratio declined from 1.99 to 1.88 over the same period. In contrast, on medium dairy farms over the same period, yields increased by $1.2 \%$, but concentrate feed input increased by less $(0.9 \%)$. Consequently, the output:input ratio remained on a slightly rising trend on medium farms. The output:input ratio increased even more on backyard farms.

Why is TFP performance so poor on larger farms? Based on our previous discussion, one of the important reasons is the reduction of fodder inputs coupled with low fodder quality. Another potential reason could be regional crop production outcomes. As previously discussed, the regional cropping production structure is positively associated with regional TFP performance. In fact, northern cropping areas (e.g., Heilongjiang, Jilin, Inner Mongolia, Hebei, Shandong, and Henan) provide better fodder sources (corn, peanut, and soybean straw) than do the southern regions.

Second, do our estimated results differ from those previously published? Previous research, based on 1990 s data, found that TFP growth was only $0.48 \%$ on specialized household dairy farms and $1.31 \%$ on commercialized dairy farms (see, for example, Rae et al., 2006). Our research suggests that TFP growth increased even faster as we moved into the new millennium, at $3.14 \%$ on backyard dairy farms and $3.20 \%$ on small dairy farms. However, some medium and many large dairy farms now appear to exhibit a rapidly declining trend, as estimated by this study.

Turning to the pattern of TFP growth, previous research suggested it was exclusively driven by technological change on both specialized household dairy farms and commercialized dairy farms in China (Rae et al., 2006). However, based on our new results, the TFP growth pattern appears to have been driven by much more diverse causes. In particular, although TFP growth was still exclusively driven by technological change on backyard dairy farms and small dairy farms, the poor TFP performance seems, by the 2000s, to be caused by the use of older technologies on medium dairy farms and large dairy farms. This probably results in a decline in the growth of technology compared with the production technology frontier. This seems particularly true for large dairy farms with a large negative growth of technology in China.

Finally, if we compare the estimates of TE levels with previous results, we see that in 2003 on national dairy farms, the average levels of TE were estimated to be 82 to $87 \%$ in 1992 and 80 to $90 \%$ based on a trans$\log$ input-oriented distance production function (Ma et al., 2007b). The average levels of TE were 68 and $64 \%$ on suburban dairy farms, based on a translog stochastic frontier production function (Ma et al., 2007a). However, new results suggest that TE has improved significantly in the 2000s. For example, Peng (2008) estimated, using a translog stochastic frontier production function over the period 2004-2006, that TE were 75 to $97 \%$ on backyard dairy farms and small dairy farms, 64 to $97 \%$ on medium dairy farms, and 71 to $98 \%$ on large dairy farms. Our new estimates of TE are somewhat different from those estimated using more aggregate data from 1990s; however, they are similar to, and on occasion higher than, those estimated by Peng (2008). This latter comparison more likely indicates that TE have significantly improved as China has moved into the new millennium.

\section{CONCLUSIONS}

In this research, we utilized production cost survey data, based upon a new classification of the dairy farming system, to study China's dairy farm productivity performance. We found that for different types of farm units, improvements in TFP arose from different combinations of $\mathrm{TC}$ and $\mathrm{TE}$, with the result that policy makers wishing to influence output will need to consider carefully how to construct and implement new policy initiatives. In particular, because of variations in crop production and fodder quality, the Guo Fu Huan Tian policy should be regional, not national.

\section{ACKNOWLEDGMENTS}

Partial financial support came from the National Natural Science Foundation (No. 70773037). 


\section{REFERENCES}

ACPCRD (Agricultural Commodity Production Cost and Return Data). 2005-2009. Agricultural Commodity Production Cost and Return Data. China Statistical Publisher, Beijing, China.

Battese, G. E., and T. J. Coelli. 1993. A stochastic frontier production function incorporating a model for technical inefficiency effects. Working Papers in Econometrics and Applied Statistics, No. 69. Department of Econometrics, University of New England, Armidale, Australia.

Battese, G. E., and T. J. Coelli. 1995. A model for technical inefficiency effects in a stochastic frontier production function for panel data. Empir. Econ. 20:325-332.

Brümmer, B., T. Glauben, and G. Thijssen. 2002. Decomposition of productivity growth using distance function: The case of dairy farms in three European countries. Am. J. Agric. Econ. 84:628644.

CDSY (China Dairy Statistical Yearbook). 2005-2009. China Dairy Statistical Yearbook. China Agricultural Publisher, Beijing, China.

Chen, B. 2012. How to improve dairy coefficient of fodder utilization. China Dairy 1:29-32. (in Chinese).

Chen, E. J., and G. H. Wan. 1997. Schooling and household farm and non-farm incomes in rural China in the 1990s. Working Paper. Chinese Economy Research Center, University of Adelaide, Australia.

Coelli, T. 1996. A guide to Frontier version 4.1: A computer program for stochastic frontier production and cost function estimation. CEPA working paper 96/07. University of New England, Armidale, Australia.

Coelli, T. J., and S. Perelman. 2000. Technical efficiency of European railways: A distance function approach. Appl. Econ. 32:19671976.

Fu, Y., and Y. Zhang. 2009. The harming of low quality fodder and bad feeding practices to dairy production. China Dairy 11:72-73. (in Chinese)

Grosskopf, S., K. Hayes, L. Taylor, and W. Weber. 1997. Budget constrained frontier measures of fiscal equality and efficiency in schooling. Rev. Econ. Stat. 79:116-124.

Hou, X. 2006. Developing modern high efficient herbage industry to support dairy industry development. China Dairy 9:21-22. (in Chinese)

Hu, C. 2007. Why had dairy cattle become beef cattle? Chin. Anim. Husb. Bull. 13:66-68. (in Chinese)

Karagiannis, G., P. Midmore, and V. Tzouvelekas. 2004. Parametric decomposition of output growth using a stochastic input distance function. Am. J. Agric. Econ. 86:1044-1057.
Khumbakar, S. C., and C. A. K. Lovell. 2000. Stochastic Frontier Analysis. Cambridge University Press, Cambridge, UK.

Li, S., C. Ping, and B. Zheng. 2007. The ways of improving feed conversion ratio to improve the dairy yield and quality. China Dairy 8:56-58. (in Chinese)

Li, Y. 2007. The new challenges faced by dairy farm production in China. Modern J. Anim. Husb. Vet. Med 8:5-6. (in Chinese)

Liu, C. 2009. Several practical issues to be solved on China's dairy industry development. Pages 46-49 in China Dairy Yearbook. China Agriculture Publisher, Beijing. (in Chinese)

Ma, H. Y., A. N. Rae, J. K. Huang, and S. Rozelle. 2007a. Enhancing productivity on suburban dairy farms in China. Agric. Econ. $37: 29-42$.

Ma, H. Y., H. C. Tang, and A. N. Rae. 2007b. An analysis of total factor productivity on China's dairy farms. Chin. Rural Econ 2:40-48. (in Chinese)

Paul, C. J. M., W. Johnson, and G. Frengley. 2000. Efficiency in New Zealand sheep and cattle farming: The impacts of regulatory reform. Rev. Econ. Stat. 82:325-337.

Peng, X. F. 2008. Analysis of technical efficiency on China's dairy farms. J. Agrotech. Econ 6:23-29. (in Chinese)

PREC (Population Resource and Environment Committee of National Consultative Committee). 2009. A report of several critical technical issues to be solved on dairy industry development. Pages 1-3 in China Dairy Yearbook. China Agriculture Publisher, Beijing, China. (in Chinese)

Rae, A. N., H. Y. Ma, J. K. Huang, and S. Rozelle. 2006. Livestock in China: Commodity-specific total factor productivity decomposition using new panel data. Am. J. Agric. Econ. 88:680-695.

Tian, W. M., and G. H. Wan. 2000. Technical efficiency and its determinants in China's grain production. J. Prod. Anal. 13:159-174.

Wang, Y. 2005. Analyses and measures in the state of transition and integration of China's dairy farm sector. Dairy Guide 7:26-27. (in Chinese)

Yang, F. 2009. Outlook of China's herbage industry development. Pages 163-164 in China Dairy Yearbook. China Agriculture Publisher, Beijing, China. (in Chinese)

Yang, Z., and S. Li. 2009. The challenge and countermeasure of China's herbage industry. China Dairy 5:60-63. (in Chinese)

Zhang, S., W. Li, Y. Zhang, J. Guo, L. Zhang, B. Wu, and B. Yang. 2010. How to move out of mistakes on dairy farming. China Dairy 2:72-73. (in Chinese) 